Moroccan J. of Pure and Appl. Anal. (MJPAA)

Volume 6(1), 2020, Pages 34-41

ISSN: Online 2351-8227 - Print 2605-6364

DOI: $10.2478 /$ mjpaa-2020-0003

\title{
Hypercyclicity of Composition Operators on Orlicz Function Spaces
}

\author{
F. JAFARI ${ }^{1}$ AND Z. KAMALI ${ }^{2}$
}

Aвstract. In this paper, we discuss the hypercyclic properties of composition operators on Orlicz function spaces. We give some different conditions under which a composition operator on Orlicz spaces is hypercyclic or not. Similarly, multiplication operators are considered. It is shown that there is no hypercyclic multiplication operator on Orlicz spaces.

Mathematics Subject Classification (2010): 47A16, 47B33, 47B38.

Key words and phrases: Composition operator, Multiplication operators, Orlicz function spaces, Hypercyclic operators.

\section{Introduction and preliminaries}

Let $(\Omega, S, \mu)$ be a complete $\sigma$-finite Borel measure space. Let denote be $L^{0}=L^{0}(\Omega, \mu)$ the set of all equivalence classes of complex valued measurable functions defined on $\Omega$. For $1 \leq p<\infty, L^{p}(\Omega, \mu)=L^{p}(\Omega, S, \mu)$ is the set of all $f \in L^{0}$ such that

$$
\|f\|_{p}=\left(\int_{\Omega}|f|^{p} d \mu\right)^{\frac{1}{P}}<\infty .
$$

Received : 31 December 2019 - Accepted: 28 February 2020.

(C) This article is published with open access by Sidi Mohamed Ben Abdallah University.

The Author(s): 1,2 Department of Mathematics, Faculty of Sciences, Shiraz Branch, Islamic Azad University, Shiraz, Iran e-mail: ${ }^{1}$ fj19413@gmail.com

e-mail: ${ }^{2}$ zkamali@shirazu.ac.ir (Corresponding Author). 
For $p=\infty, L^{\infty}(\Omega, \mu)$ is the set of all equivalence classes of essentially bounded measurable functions $f: \Omega \rightarrow \mathbb{C}$ with the norm

$$
\|f\|_{\infty}=\operatorname{ess} \sup _{\Omega}|f|:=\inf \{\alpha \in \mathbb{C}: \mu(\{x \in \Omega:|f(x)|>\alpha\})=0\} .
$$

Let $\Phi:[0, \infty) \rightarrow[0, \infty)$ be an Orlicz function, i.e. continuous, convex, increasing function, $\Phi(0)=0, \lim _{x \rightarrow \infty} \Phi(x)=\infty$ and $\lim _{x \rightarrow \infty} \frac{\Phi(x)}{x}=\infty\left(\operatorname{resp} . \lim _{x \rightarrow 0} \frac{\Phi(x)}{x}=0\right)$.

Then, we define the Orlicz spaces as follows

$$
L^{\Phi}(\Omega, \mu)=\left\{f \in L^{0}: \int_{\Omega} \Phi(\lambda|f|) d \mu<\infty \text { for some } \lambda>0\right\} .
$$

This space endowed with the Luxemburg norm, which is defined by

$$
\|f\|_{\Phi}=\inf \left\{\lambda>0: \int_{\Omega} \Phi\left(\frac{|f|}{\lambda}\right) d \mu \leq 1\right\} .
$$

is a Banach space.

Now, we give some notations and we briefly review certain properties which will be used later.

- If $\Phi(t)=t^{p}$, then $L^{\Phi}(\Omega, \mu)=L^{p}(\Omega, \mu)$.

- If $A \in S$ and $0<\mu(A)<\infty$, then

$$
\left\|\chi_{A}\right\|_{\Phi}=\frac{1}{\Phi^{-1}\left(\frac{1}{\mu(A)}\right)}
$$

where $\Phi^{-1}(t)=\inf \{r>0: \Phi(r)>t\}$ is the right-inverse of $\Phi$.

- The Orlicz function $\Phi$ is called to satisfy the $\Delta_{2}$-condition, written $\Phi \in \Delta_{2}$, if there are constants $k>0$ and $t_{0} \geq 0$ such that $\Phi(2 t) \leq k \Phi(t)$, for all $t \geq t_{0}$.

- The measurable transformation $T: \Omega \rightarrow \Omega$ is said to be measure preserving if $\mu\left(T^{-1}(A)\right)=$ $\mu(A)$ for every $A \in S$.

- A subset $A \in S$ is said to be an atom with $\mu(A)>0$, if for every $E \in S$ such that $E \subset A$, then $\mu(E)=0$ or $\mu(E)=\mu(A)$.

- A measurable transformation $T: \Omega \rightarrow \Omega$ is called non-singular if

$$
\mu T^{-1}(A)=\mu\left(T^{-1}(A)\right)=0,
$$

whenever $\mu(A)=0$ for all $A \in S$. This condition means that the measure $\mu T^{-1}$ is absolutely continuous with respect to $\mu$ (It is usually denoted by $\mu T^{-1} \ll \mu$ ). Then the Radon-Nikodym theorem assures the existence of a unique non-negative Borel measurable function $h \in L^{1}(\Omega, \mu)$ on $\Omega$ such that

$$
\mu T^{-1}(A)=\int_{A} h(t) d \mu(t) \text { for all } A \in S .
$$

In this case $h$ is said to be the Radon-Nikodym derivative and denoted by $\frac{d \mu T^{-1}}{d \mu}$.

- A non-singular measurable transformation $T$ induces a well-defined composition operator $C_{T}$ from $L^{\Phi}(\mu)$ into itself defined by $C_{T} f(x)=f(T(x))$ for $x \in \Omega$ and $f \in L^{\Phi}(\Omega, \mu)$. Note that if $T$ is not non-singular transformation, then $C_{T}$ is not well-defined (see for instance [20, page 18]). 
- Let $u: \Omega \rightarrow \mathbb{C}$ be a function such that $u$.f $\in L^{\Phi}(\Omega, \mu)$ for every $f \in L^{\Phi}(\Omega, \mu)$. If $u$ is a function in $L^{\infty}(\Omega, \mu)$, then the multiplication operator $M_{u}$ from $L^{\Phi}(\Omega, \mu)$ into itself defined by $M_{u} f=u . f$ for any $f \in L^{\Phi}(\Omega, \mu)$ is a bounded operator (see [13]).

In the literature corresponding to the Orlicz spaces, the basic properties of these spaces were considered by Rao and Ren in [17]. Cui, Hudzic, Kumar and Maligranda [7] and Komar [15] proved some properties of compositon operators on Orlicz spaces. In [13] Komal and Gupta established certain properties of multiplication operators on Orlicz spaces. Finally, Chen and $\mathrm{Du}$ [5] presented new characterizations of disjoint topological transitivity on Orlicz spaces.

Next, a bounded linear operator $T$ on Banach space $X$ is (weakly) hypercyclic if there exists a vector $x \in X$ such that the orbit of $x$ under $T$ defined by

$$
\operatorname{Orb}(T, x):=\left\{T^{n} x: n \in \mathbb{N} \cup\{0\}\right\}
$$

is (weakly)dense in $X$. Every such vector $x$ is called (weakly) hypercyclic vector for $T$.

In recent years, great attention has been focused on the study of hypercyclic operators. The first example of a hypercyclic operator is $\lambda B$ for $|\lambda|>1, \lambda \in \mathbb{C}$ on the space $l^{2}(\mathbb{N})$, where $B$ is the backward shifts. For more details, we refer the interested reader to $[2,18]$, and the references therein.

\section{Hypercyclic Composition Operators on $L^{\Phi}(\Omega, \mu)$}

In this section, we discuss about hypercyclic properties of composition operators on Orlicz function spaces.

Lemma 2.1. If $T$ is a non-singular transformation and $\frac{d \mu T^{-1}}{d \mu}=h$, then for all $n \in \mathbb{N}, T^{n}$ is also non-singular transformation and $\frac{d \mu T^{-n}}{d \mu}=\prod_{j=0}^{n-1}$ ho $T^{-j}$. Moreover, if $T$ is invertible and its inverse is also non-singular transformation, then for all $n \in \mathbb{N}, \frac{d \mu T^{n}}{d \mu}=\left(\prod_{j=1}^{n} h \circ T^{j}\right)^{-1}$.

Proof. We use induction on $n$. This is true for $n=1$. Suppose it is true for $n$, we prove for $n+1$ is true. Let $E \in S$ and $\mu(E)<\infty$, so

$$
\mu T^{-(n+1)}(E)=\mu T^{-n}\left(T^{-1}(E)\right)=\int_{T^{-1}(E)} \prod_{j=0}^{n-1} h o T^{-j} d \mu
$$

Using a change of variable, we get

$$
\mu T^{-(n+1)}(E)=\int_{E} \prod_{j=1}^{n} h o T^{-j} d \mu T^{-1}=\int_{E} h \prod_{j=1}^{n} h o T^{-j} d \mu=\int_{E} \prod_{j=0}^{n} h o T^{-j} d \mu
$$

By uniquness of Radon-Nikodym derivative theorem, we have

$$
\frac{d \mu T^{-n}}{d \mu}=\prod_{j=0}^{n-1} h o T^{-j}
$$


For $E \in S$, we obtain

$$
\int_{E} h(T(x)) d \mu T(x)=\int_{T(E)} h(r) d \mu(r)=\mu\left(T^{-1}(T(E))=\mu(E) .\right.
$$

So

$$
\frac{d \mu}{d \mu T}=h o T \text { or } \frac{d \mu T}{d \mu}=\frac{1}{h o T} .
$$

For the rest of proof we follow the same steps as before.

In [12] the authors presented the hypercyclicity criterion. This criterion was independently improved by Gethner and Shapiro [8]. Eventually, the most general example of this criterion was expressed by Bés [3].

Hyperciclicity Criterion (see [2]). Suppose that $T$ is a continuous linear operator on a separable Banach space $X$, for which the sequence of non-negative powers $\left(T^{n}\right)$ tends pointwise to zero on a dense subset of $X$. Suppose further that there is a (possibly different) dense subset $Y$ of $X$, and a (possibly discontinuous) map $S: Y \rightarrow Y$ such that $T S=$ identity on $Y$, and $\left(S^{n}\right)$ tends pointwise to zero on $Y$, then $T$ is hypercyclic.

Remark 1. In [16] Kufner, John and Fucik proved that $L^{\Phi}(\Omega, \mu)$ is a separable Banach space, if $\Phi$ satisfies the $\Delta_{2}$-condition. Since hypercyclicity has sense only if the underlying space is separable, then we consider only the case where $\Phi$ satisfies the $\Delta_{2}$-condition.

Theorem 2.1. Let $T: \Omega \rightarrow \Omega$ be a non-singular measurable transformation, its inverse is measurable and non-singular transformation. Assume that $\Phi:[0, \infty) \rightarrow[0, \infty)$ be an Orlicz function such that $\Phi$ satisfies the $\Delta_{2}$-condition and $C_{T} \in B\left(L^{\Phi}(\Omega, \mu)\right)$. If

$$
\prod_{j=0}^{n-1} h o T^{-j} \rightarrow 0 \text { a.e as } n \rightarrow \infty \text { and }\left(\prod_{j=1}^{n} h o T^{j}\right)^{-1} \rightarrow 0 \text {, a.e., as } n \rightarrow \infty
$$

Then $C_{T}$ is hypercyclic on $L^{\Phi}(\Omega, \mu)$.

Proof. For $A \in S$ and $0<\mu(A)<\infty$, we have

$$
\left\|C_{T}^{n} \chi_{A}\right\|_{\Phi}=\left\|\chi_{T^{-n}(A)}\right\|_{\Phi}=\frac{1}{\Phi^{-1}\left(\frac{1}{\mu\left(T^{-n}(A)\right)}\right)}
$$

Therefore, by Lemma 2.1, we get

$$
\left\|C_{T}^{n} \chi_{A}\right\|_{\Phi}=\frac{1}{\Phi^{-1}\left(\frac{1}{\int_{A} \prod_{j=0}^{n-1} h o T^{-j}(t) d \mu(t)}\right)}
$$

Since $\Phi$ is an increasing function, so by [9, Lemma 2.3.9], $\Phi^{-1}$ is continuous and $\Phi \in \Delta_{2}$ implies that $\lim _{t \rightarrow \infty} \Phi^{-1}(t)=\infty$. Consider the fact that $\prod_{j=0}^{n-1} h o T^{-j}$ is Rodon-Nykodym derivative and $\mu$ is $\sigma$-finite measure, hence as in [19], we obtain

$$
\int_{A} \prod_{j=0}^{n-1} h o T^{-j}(t) d \mu(t)<\infty
$$


Then by Lebesgue Convergence theorem, we have

$$
\left\|C_{T}^{n} \chi_{A}\right\|_{\Phi} \rightarrow 0 \text { as } n \rightarrow \infty \text {. }
$$

So, for all simple functions $s$, it follows

$$
\left\|C_{T}^{n} s\right\|_{\Phi} \rightarrow 0 \text { as } n \rightarrow \infty
$$

Similarly, we find that

$$
\left\|C_{T}^{-n}\right\|_{\Phi} \rightarrow 0 \text { as } n \rightarrow \infty .
$$

Since simple functions are dense in $L^{\Phi}(\Omega, \mu)$, thus by Hypercyclic Criterion, $C_{T}$ is hypercyclic on $L^{\Phi}(\Omega, \mu)$.

Example 2.1. Let $\Omega=[0,1]$ and $\mu$ be a Lebesgue measure on $[0,1]$. Consider $T(x)=1-\sqrt{1-x}$. So $T$ be a non-singular transformation and $h=2(1-x)$ by [10], we have:

$$
\prod_{j=0}^{n-1} h o T^{-j}(x)=\prod_{j=0}^{n-1} 2(1-x)^{2^{j}}=2^{n} \cdot(1-x)^{2^{n}-1},
$$

and

Since

$$
\prod_{j=1}^{n} h o T^{j}(x)=\prod_{j=1}^{n} 2 \sqrt[2^{n}]{1-x}=2^{n} \cdot(1-x)^{1-\left(\frac{1}{2}\right)^{n+1}} .
$$

$$
\lim _{n \rightarrow \infty} 2^{n} \cdot(1-x)^{2^{n}-1}=0 \text { and } \lim _{n \rightarrow \infty}\left(2^{n} \cdot(1-x)^{1-\left(\frac{1}{2}\right)^{n+1}}\right)^{-1}=0 .
$$

Thus, by Theorem 2.1, $C_{T}$ is hypercyclic.

Next, For an Orlicz function $\Phi$ define

$$
b_{\Phi}=\sup \{u>0: \Phi(u)<\infty\} \text { and } a_{\Phi}=\inf \{u>0: \Phi(u)>0\} .
$$

Clearly, $0 \leq a_{\Phi} \leq b_{\Phi} \leq \infty$.

Theorem 2.2. If $0<a_{\Phi} \leq b_{\Phi}<\infty$, then there is no weakly hypercyclic composition operator on $L^{\Phi}(\Omega, \mu)$.

Proof. If $0<a_{\Phi}=b_{\Phi}<\infty$, then

$$
\Phi(t)=\left\{\begin{array}{ccc}
0 & \text { if } & 0 \leq t \leq a_{\Phi} \\
\infty & \text { if } & a_{\Phi}<t<\infty .
\end{array}\right.
$$

Let $f \in L^{\Phi}(\Omega, \mu)$, So there exists $\lambda>0$ such that $\int_{\Omega} \Phi(\lambda|f|) d \mu<\infty$. By the definition of $\Phi$, we must have $0<\lambda|f| \leq a_{\Phi}$, that is, $|f| \leq \frac{a_{\Phi}}{\lambda}$. This means $f \in L^{\infty}(\Omega, \mu)$, and $\|f\|_{\Phi} \geq \frac{\|f\|_{\infty}}{a_{\Phi}}$. Now, suppose $f \in L^{\infty}(\Omega, \mu)$, so there is $\lambda>0$ such that $\lambda|f| \in\left[0, a_{\Phi}\right]$, it follows that $\int_{\Omega} \Phi(\lambda|f|) d \mu=$ 0 , and $f \in L^{\Phi}(\Omega, \mu)$. Thus $\|f\|_{\Phi} \leq \frac{\|f\|_{\infty}}{a_{\Phi}}$. Hence, in this case, $L^{\Phi}(\Omega, \mu)=L^{\infty}(\Omega, \mu)$ with $\|f\|_{\infty}=a_{\Phi}\|f\|_{\Phi}$.

Next, suppose that $0<a_{\Phi}<b_{\Phi}<\infty$. Choose $\epsilon>0$ such that $0<\epsilon<a_{\Phi}$ and $\Phi(\epsilon)=0$. Since $(\Omega, S, \mu)$ be a $\sigma$-finite measure space, there exists disjoint subsets $\Omega_{n}$ of $\Omega$ with $\mu\left(\Omega_{n}\right)<\infty$ 
such that $\Omega=\cup_{n=1}^{\infty} \Omega_{n}$. Suppose that $f \in L^{\infty}(\Omega, \mu)$, so there is $\lambda>0$ such that $\frac{\|f\|_{\infty}}{\lambda} \leq \epsilon$. Since $\Phi$ be an increasing function, we have

$$
\int_{\Omega_{n}} \Phi\left(\frac{|f|}{\lambda}\right) d \mu \leq \Phi(\epsilon) \mu\left(\Omega_{n}\right)=0 .
$$

So, for all $n \in \mathbb{N}$ we obtain $\int_{\Omega_{n}} \Phi\left(\frac{|f|}{\lambda}\right) d \mu=0$. As $\int_{\Omega} \Phi\left(\frac{|f|}{\lambda}\right) d \mu=\sum_{n=1}^{\infty} \int_{\Omega_{n}} \Phi\left(\frac{|f|}{\lambda}\right) d \mu$. Then $\int_{\Omega} \Phi\left(\frac{|f|}{\lambda}\right) d \mu=0$, thus $f \in L^{\Phi}(\Omega, \mu), L^{\Phi}(\Omega, \mu) \subseteq L^{\infty}(\Omega, \mu)$ and $\|f\|_{\Phi} \leq \frac{\|f\|_{\infty}}{\epsilon}$.

Conversely, suppose that $f \in L^{\Phi}(\Omega, \mu)$. So there exists $\lambda>0$ such that $\int_{\Omega} \Phi(\lambda|f|) d \mu \leq \infty$. For all $n \in \mathbb{N}$, we define

$$
A_{n}=\{x \in \Omega:|f(x)|>n\}, \quad B_{n}=\{x \in \Omega: \Phi(\lambda|f(x)|)>\Phi(\lambda n)\} .
$$

Since $\Phi$ be an increasing function, we have $A_{n} \subseteq B_{n}$. By assumption we have $b_{\Phi}<\infty$, so there exists $x_{0} \neq 0, n_{0} \in \mathbb{N}$ such that $\Phi\left(x_{0}\right)=\infty$ and $\lambda n_{0}>x_{0}$. Consequently, $\Phi\left(\lambda n_{0}\right)=\infty$ and

$$
\infty>\int_{\Omega} \Phi(\lambda|f|) d \mu \geq \int_{B_{n_{0}}} \Phi(\lambda|f|) d \mu \geq \int_{B_{n_{0}}} \Phi\left(\lambda n_{0}\right) d \mu .
$$

Since $\mu\left(A_{n_{0}}\right) \leq \mu\left(B_{n_{0}}\right), \mu\left(A_{n_{0}}\right)=0, f \in L^{\infty}(\Omega, \mu)$ and $\|f\|_{\infty} \leq n_{0}$. Hence, $\mu\left(B_{n_{0}}\right)=0$. Thus in this case $L^{\Phi}(\Omega, \mu)=L^{\infty}(\Omega, \mu)$ and $\frac{\|f\|_{\infty}}{n_{0}} \leq\|f\|_{\phi} \leq \frac{\|f\|_{\infty}}{\epsilon}$. On the other hand for all $f \in$ $L^{\infty}(\Omega, \mu)$, we have $\|f o T\|_{\infty} \leq\|f\|_{\infty}$, so $\left\|C_{T}\right\|_{\infty} \leq 1$, and $C_{T}$ cannot be weakly hypercyclic on $L^{\infty}(\Omega, \mu)$ and also on $L^{\Phi}(\Omega, \mu)$.

Proposition 2.1. If $C_{T}$ is (weakly) hypercyclic on $L^{\Phi}(\Omega, \mu)$, then:

a) $T^{-1}(S)=$ S i.e. $S=\left\{T^{-1}(A): A \in S\right\}$.

b) $T$ is not a non-singular measure-preserving transformation.

c) $L^{\Phi}\left(X_{\varepsilon}, \mu T^{-1}\right)$ is infinite dimensional for each $\varepsilon>0$, where $X_{\varepsilon}=\left\{x \in \Omega: \frac{d \mu T^{-1}}{d \mu}(x) \geq \varepsilon\right\}$.

d) $N(h, \varepsilon)=\left\{x \in \Omega: h=\frac{d \mu T^{-1}}{d \mu}(x)>\varepsilon\right\}$ consists of infinitely many atoms for each $\varepsilon$.

d) $h \geq 1$.

Proof. a) Suppose $T^{-1}(S) \neq S$. By [14, Corollary 4.5], since $C_{T}$ has not dense range, so is not (weakly) hypercyclic.

b) Let $T$ be a non-singular measure-preserving transformation, then by [14, Corollary 4.8$] C_{T}$ is an isometry. Thus, by [1, Corollary 3.2] $C_{T}$ can not be (weakly) hypercyclic on $L^{\Phi}(\Omega, \mu)$.

c) Suppose that $L^{\Phi}\left(X_{\varepsilon}, \mu T^{-1}\right)$ is finite dimensional for each $\varepsilon>0$, then by [14, Theorem 3.1] $C_{T}$ is compact. Thus $C_{T}$ is not (weakly)hypercyclic.

d) Suppose that $N(h, \varepsilon)$ consists of finitely many atoms, so by [10, Proposition 2.1] $C_{T}$ is compat, therefore $C_{T}$ is not (weakly) hypercyclic on $L^{\Phi}(\Omega, \mu)$.

e) Let $h<1$, so by [7, Remark 3], $\left\|C_{T}\right\|_{\Phi} \leq 1$. Hence $C_{T}$ can not be (weakly) hypercyclic on $L^{\Phi}(\Omega, \mu)$. 


\section{Hypercyclic Muitiplication Operators on $L^{\Phi}(\Omega, \mu)$}

In this section, we show that there is no hypercyclic multiplication operators on Orlicz function spaces. For this we use the same method as the one used by that authors in [6].

Theorem 3.1. Let $(\Omega, S, \mu)$ be a regular $\sigma$-finite measure space. If $M_{u} \in B\left(L^{\Phi}(\Omega, \mu)\right)$, then $M_{u}$ is not hypercyclic on $L^{\Phi}(\Omega, \mu)$.

Proof. Since $\left\|M_{u}\right\|_{\Phi}=\|u\|_{\infty}$, we have two cases, $\|u\|_{\infty} \leq 1$ and $\|u\|_{\infty}>1$. We prove that $M_{u}$ is not hypercyclic in this both cases. If $\|u\|_{\infty} \leq 1$, then $\left\|M_{u}\right\|_{\Phi} \leq 1$. So $M_{u}$ is not hypercyclic. Now, we suppose that $\|u\|_{\infty}>1$. Since $\mu$ is regular, therefore $\mu(\Omega)=\sup \{\mu(K)$ : $K$ is compact, $K \subseteq \Omega$, so there is a compact set $K \subset \Omega$ such that $\mu(K)>0$. On the other hand, $\lim _{x \rightarrow \infty} \Phi(x)=\infty$, there exists $\varepsilon>0$ such that $\Phi\left(\frac{1}{\varepsilon}\right)>\frac{2}{\mu(K)}$ or $\mu(K)>\frac{2}{\Phi\left(\frac{1}{\varepsilon}\right)}$. Moreover, $u$ is continuous, so we may assume $u(x)>1$ for all $x \in K$. Since the hypercyclic vectors for $M_{u}$ are dense in $L^{\Phi}(\Omega, \mu)$, then there exists a hypercyclic vector $f$ for $M_{u}$ such that for sufficiently large $n \in \mathbb{N}$, we have:

$$
\left\|f-2 \chi_{K}\right\|_{\Phi}<\varepsilon \text { and }\left\|M_{u}^{n} f\right\|_{\Phi}<\varepsilon .
$$

Let $W=\{x \in K:|f(x)|<1\}$ and suppose $\mu(W) \neq 0$. Then

$$
\varepsilon>\left\|f-2 \chi_{K}\right\|_{\Phi} \geq\left\|\chi_{W} \cdot\left(f-2 \chi_{K}\right)\right\|_{\Phi} \geq\left\|\chi_{W}\right\|_{\Phi}=\frac{1}{\Phi^{-1}\left(\frac{1}{\mu(W)}\right)} .
$$

Therefore in the both cases $\mu(W)<\frac{1}{\Phi\left(\frac{1}{\varepsilon}\right)}$. On the other hand, suppose $Q=K-W$, and $\mu(Q) \neq 0$.

$$
\varepsilon>\left\|M_{u}^{n} f\right\|_{\Phi} \geq\left\|\chi_{Q} \cdot\left(u^{n} f\right)\right\|_{\Phi} \geq\left\|\chi_{Q}\right\|_{\Phi}=\frac{1}{\Phi^{-1}\left(\frac{1}{\mu(Q)}\right)} .
$$

Hence $\mu(Q)=\mu(K-W)=\frac{1}{\Phi\left(\frac{1}{\varepsilon}\right)}$. So in all cases $\mu(K)=\mu(W)+\mu(Q)<\frac{2}{\Phi\left(\frac{1}{\varepsilon}\right)}$, which is a contradiction.

\section{References}

[1] F. Bayart, m-isometries on Banach spaces, Math.Nachr., 284 (17-18) (2011), 2141-2147.

[2] F. Bayart and E. Atheron, Dynamics of linear operators, Cambridge Tracts in Mathematics 179, (2009).

[3] J. P. Bés, Three problem's on hypercyclic operators, Ph.D. thesis, Bowling Green State University, (1998).

[4] P. S. Bourdon and J. H. Shapiro, Cyclic phenomena for composition operators, Memoirs Amer. Math. Soc., 596 (1997). ISSN 0065-9266

[5] C. C. Chen and W. S. Du, Some characterizations of disjoint topological transitivity on orlicz spaces, Journal of Inequalities and Applications, 88(1) (2018).

[6] C. Chen, C. -H. Chu, Hypercyclic weighted translation on groups, Proc. Amer. Math. Soc., 139(8) (2011), 2839-2846.

[7] Y. Cui, H. Hudzic, R. Kumar and R. Maligranda, Composition operators in orlicz spaces, J. Aust. Math. Soc. 76(2) (2004), 189-206. 
[8] R. M. Gethner and J. H. Shapiro, Universal vectors for operators on spaces of holomorphic functions, Proc. Amer. Math. Soc., 100(2) (1987), 281-288. ISSN 0019-3577.

[9] P. Harjulehto and P. Hasto, Orlicz spaces and Generalized Orlicz spaces, Lecture Notes in Mathematics, Springer International Publishing, (2019).

[10] M. R. Jabbarzadeh, Essential norm of composition operator on orlicz space, Turk. J. Math, 34 (2010), 537-542.

[11] M. R. Jabbarzadeh and Y. Estaremi, essential norm of subtitution operators on $L^{p}-$ spaces, Indian J Pure Appl Math, 43(3) (2012), 263-278. ISSN 0019-5588

[12] C. Kitai, Invariant closed sets for linear operators, Thesis, Univ of Toronto, (1982).

[13] B. S. Komal, S. Gupta, Multiplication operators between orlicz spaces, Integral Equations Operator Theory, 41 (2001), 324-331.

[14] B. S. Komal, S. Gupta, Composition operators on orlicz spaces, Indian J. Pure Appl. Math., 32(7) (2001), 11171122.

[15] R. Komar, Composition operators on orlicz spaces, Integral Equ. Oper. Theory, 29(1) (1997), 17-22.

[16] A. Kufner, O. John and S. Fuick, Function Spaces, Mechanics: Analysis, Springer Netherlands, (1977).

[17] M. M. Rao and Z. D. Ren, Theory of Orlicz spaces, Marcel Dekker, Inc. New York, Basel and Hong Kong, (1991).

[18] S. Rolewicz, On orbits of elements, Studia. Math., 32 (1969), 17-22.

[19] W. Rudin, Real and complex analysis, MacGraw-Hill Book Company, 1987.

[20] R. K. Singh and J. S. Manhas, Composition Operators on Function Spaces, Mathematics Studies NorthHolland, Vol.(179), (1993). 